\title{
Using UV-Vis. linear dichroism to study the orientation of molecular probes and biomolecules in lipidic membranes
}

\author{
M.A.R.B. Castanho ${ }^{\mathrm{a}, \mathrm{b}, *}$, S. Lopes ${ }^{\mathrm{b}}$ and M. Fernandes ${ }^{\mathrm{c}}$

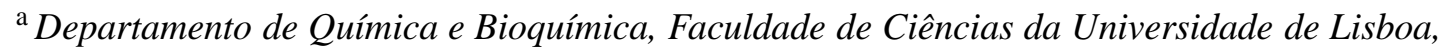 \\ Campo Grande C8, 1749-016 Lisboa, Portugal \\ ${ }^{\mathrm{b}}$ Centro de Química Física Molecular, Complexo I, Instituto Superior Técnico, Av. Rovisco Pais, \\ 1049-001 Lisboa, Portugal \\ ${ }^{\mathrm{c}}$ Departamento de Química Física, Universidad de Murcia, 30071 Murcia, Spain
}

\begin{abstract}
Linear dichroism methodologies are based on the different interaction of molecules with linearly polarized light depending on their orientation. Theoretical predictions are used to conclude on the orientation of selected molecules relative to their neighbours (usually an organized matrix). In the specific case of linear dichroism methodologies applied to data obtained from UV-Vis. spectroscopic techniques, the orientational distribution function of the chromophores' electronic transition moment can be calculated and converted to the molecular axis distribution function. In this paper, the orientation of molecular probes and biomolecules in model systems of biomembranes (lipidic matrixes) is explored and illustrated using common membrane probes (trans-parinaric acid, a cyanine and laurdan) and polyene antibiotics (Amphotericin B and Nystatin). The emphasis is on the technique and methodologies themselves, rather than the scientific impact of the attained distributions. The main addressed items are: (1) How to adapt a common UV-Vis. spectrophotometer and spectrofluorimeter to perform linear dicrhoism experiments?, (2) Sample preparation, and (3) Data analysis (including artefacts corrections).
\end{abstract}

\section{Introduction}

Few methods are able to reveal the orientation of molecules inserted in ordered matrixes, e.g., biological membranes. Nevertheless, molecular orientation in biological membranes is a key-issue to understand immune responses or cellular communication, for instance (receptor/ligand interactions, in a broad sense). Spectroscopic techniques such as electron paramagnetic resonance [58], infra-red [15] or nuclear magnetic resonance [22] can be used to study molecular orientation in lipidic bilayers. Fourier transform infra-red spectroscopy has been widely used $[10,16]$. However, as it is sensitive to the orientation of specific chemical bonds relative to the lipidic bilayer plane, deducing the global molecular orientation may be quite complex. This task is simplified when UV-Vis. spectroscopic techniques are used because the transition moment "covers" the entire chromophoric group. Moreover, we have combined spectroscopic and Brownian dynamics simulation data to conclude on the overall molecular orientation. The strengths, weaknesses, instrumental needs and theoretical framework of UV-Vis. linear dichroism are discussed in this paper. A broader picture of linear dichroism can be obtained from [4] and [18].

\footnotetext{
*Corresponding author. Tel.: +351 21 7500931; Fax +351 21 7500088; E-mail: castanho@ fc.ul.pt.
} 


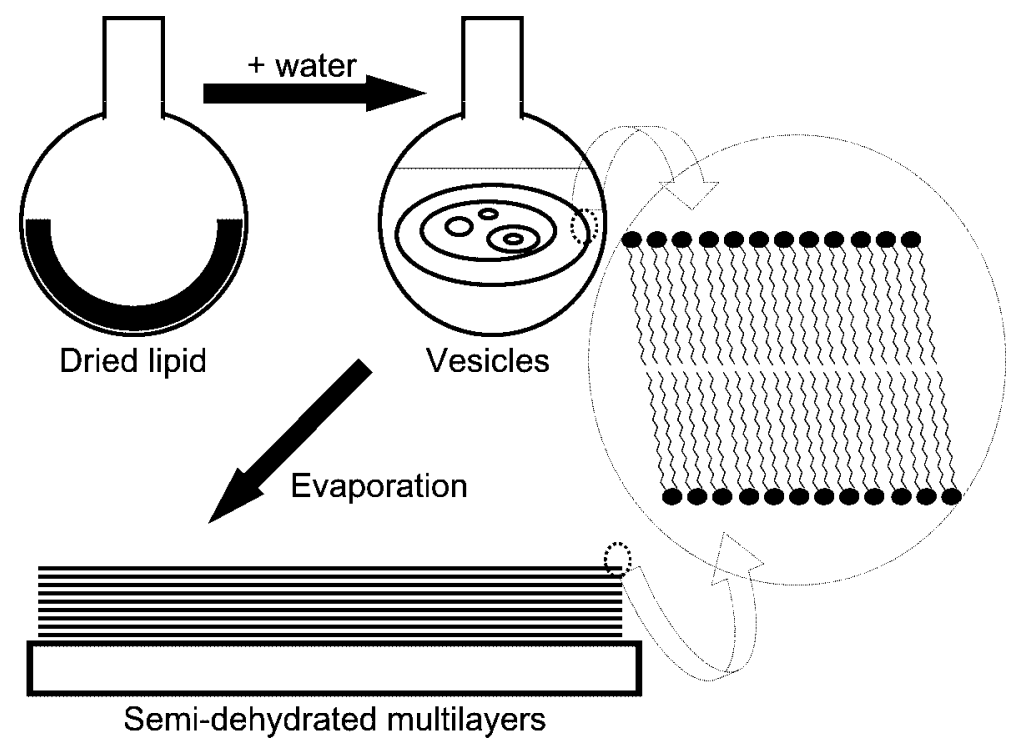

Fig. 1. Brief outline of sample preparation, highlighting the fact that lipidic bilayers are microscopically anisotropic, whether forming vesicles (macroscopically isotropic) or planar supported bilayers stacks (macroscopically anisotropic) semi-dehydrated multilayers. Sample preparation begins by preparing a pure lipidic film on a round bottom flask (evaporated chloroform solution; total evaporation is achieved under vacuum), followed by full hydration (shaking and heating/cooling cycles lead to vesicles formation) and, finally, semi-dehydration on a quartz slide (see Fig. 8). Hydration water is kept in the sample and multibilayers (tens to hundreds of stacked bilayers) are spontaneously formed.

\section{Orientational distribution functions: theoretical background, experimental design and data analysis}

Phospholipidic membrane systems can be either macroscopically and microscopically anisotropic (e.g., multibilayers supported in a hard surface) or, microscopically anisotropic and macroscopically isotropic (e.g., suspended vesicles; Fig. 1). At the molecular level, phospholipidic membranes are always anisotropic. Our goal is to know the orientation of molecular probes and biomolecules when inserted in this anisotropic medium. Thus, the orientation reference axis should be related to the membrane system (not a laboratory frame). The membrane symmetry axis is perpendicular to its surface. Even if the lipidic chains are tilted [51], the symmetry axis is not altered; the angle between acyl chains and the membrane normal is constant but the distribution around it is random in the whole macroscopic sampled volume because the membrane is composed of many patches with their own orientation (in the scheme depicted in Fig. 2, $\psi$ is constant and $\phi$ is random for lipid chains orientations). Therefore, distribution functions are considered relative to the membranes' surface normal (system director).

In the simplest conceivable case, cylindrical molecules are considered so that molecular orientation determination is, in practice, finding out the angle $\psi$ between the long molecular axis and the system director (a more general approach can be found in [6] and [22]). For a molecular population in equilibrium, a distribution has to be considered (e.g., according to the Boltzmann potential). The orientational density probability function of such distribution, i.e., the probability of finding any of the molecules of that population in the range $\psi$ to $\psi+\mathrm{d} \psi$, is our main goal. This function is a product of a single-molecule term, $f(\psi)$, and a term that accounts for the increase of the spherical crown surface defined by $\psi+\mathrm{d} \psi$ as $\psi$ increases $(\sin (\psi))$. Thus, $f(\psi) \sin (\psi)$ is the target of one's quest. 


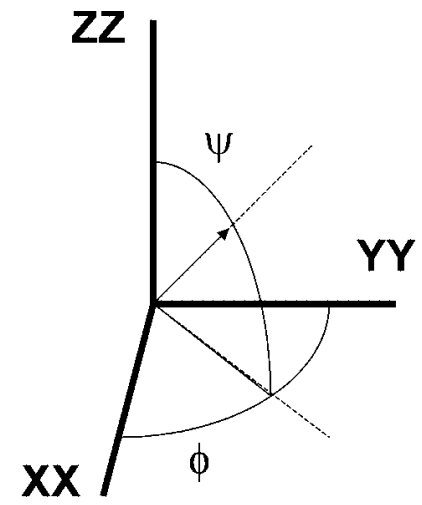

Fig. 2. The orientation of a cylinder is fully described by angles $\psi$ and $\phi$.

$f(\psi)$ can generally be described as a Legendre polynomial series [22,37].

$$
f(\psi)=\sum_{L \text { even }} \frac{1}{2}(2 L+1)\left\langle P_{L}\right\rangle P_{L}(\cos \psi)
$$

so that

$$
\int_{0}^{\pi} \sin (\psi) f(\psi) \mathrm{d} \psi=1
$$

while

$$
\left\langle P_{L}\right\rangle=\int_{-1}^{1} P_{L}(\cos \psi) f(\psi) \mathrm{d}(\cos \psi) .
$$

$\left(P_{L}(\cos \psi)\right.$ are Legendre polynomials; $\left\langle P_{L}\right\rangle$ is the ensemble-average of $P_{L}(\cos \psi)$ and is referred to as the $L$ th rank order parameter, when $L \neq 0$.)

As $f(\psi)$ is symmetrical $(f(\psi)=f(-\psi)$ and $f(\psi)=f(\pi-\psi)),\left\langle P_{L}\right\rangle=0$ if $L$ is odd. Therefore:

$$
\begin{aligned}
& f(\psi)=\frac{1}{2}+\frac{5}{2}\left\langle P_{2}\right\rangle P_{2}(\cos \psi)+\frac{9}{2}\left\langle P_{4}\right\rangle P_{4}(\cos \psi)+\cdots \\
& \quad P_{2}(\cos \psi)=\frac{3 \cos ^{2} \psi-1}{2} \quad \text { and } \quad P_{4}(\cos \psi)=\frac{35 \cos ^{4} \psi-30 \cos ^{2} \psi+3}{8},
\end{aligned}
$$

because $P_{0}(\cos \psi)=1$. For an isotropic system, $\left\langle P_{L}\right\rangle=0$ if $L \geqslant 2$ and $f(\psi)=1 / 2$. For perfectly aligned molecules, $\left\langle P_{L}\right\rangle=1$ if $L \geqslant 2$ and $f(\psi)=\delta(\cos \psi-1)$ ( $\delta$ is Dirac's delta function). Intermediate distributions are characterized by their peculiar set of $\left\langle P_{2}\right\rangle,\left\langle P_{4}\right\rangle,\left\langle P_{6},\right\rangle, \ldots$ However, only $\left\langle P_{2}\right\rangle$ and $\left\langle P_{4}\right\rangle$ can be known from experiment. Thus, the quest for $f(\psi) \sin (\psi)$ resumes to two steps: (1) $\left\langle P_{2}\right\rangle$ and $\left\langle P_{4}\right\rangle$ determination, and (2) finding an approximated function for $f(\psi) \sin (\psi)$ from $\left\langle P_{2}\right\rangle$ and $\left\langle P_{4}\right\rangle$ only.

2.1. Calculation of $\left\langle P_{2}\right\rangle$ and $\left\langle P_{4}\right\rangle$ from $U V$-Vis. spectroscopy in aligned samples (UV-Vis. linear dichroism, $L D)$

Johansson and co-workers developed the formalism that describes the dependence of linearly polarized light absorption in macroscopically aligned membranes [3,29-31,33]. Considering the instrumental set- 


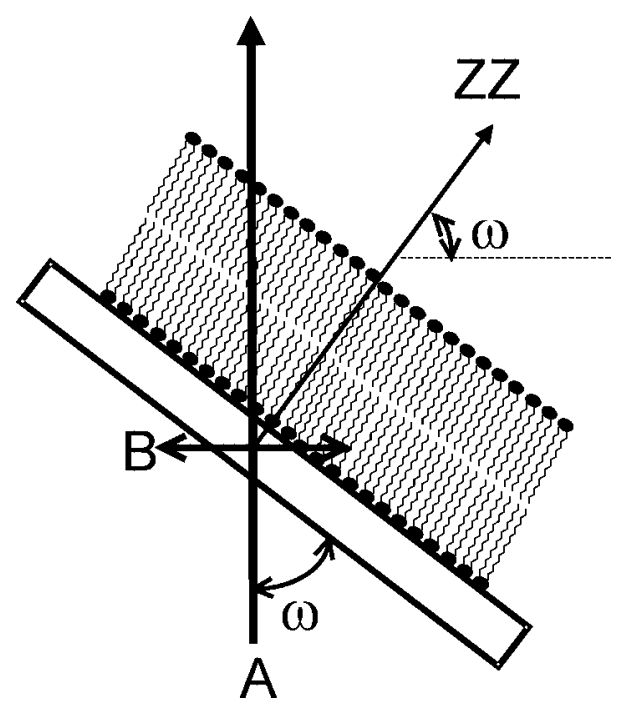

Fig. 3. Schematic representation of the experimental set-up for UV-Vis. absorption LD studies in lipidic membranes (not drawn to scale). The incident beam (A), with polarization B ("horizontal" in the lab frame), impinges on the quartz slide and supported bilayers (only one bilayer is represented for the sake of clarity). The angle between them $(\omega)$ equals the one formed by the system director (ZZ) and the light polarization (B). $\omega$ variation is accomplished by rotation of the quartz slide around the vertical axis where $\mathrm{A}$ and $\mathrm{B}$ intersect.

up depicted in Fig. 3, absorption variation with $\omega, A_{\omega}$ ( $\omega$ is the angle formed by the polarization direction relative to the system director), is given by:

$$
\frac{\sin (\omega) A_{\omega}}{A_{\omega=\pi / 2}}=1+\frac{3\left\langle P_{2}\right\rangle}{\left(1-\left\langle P_{2}\right\rangle\right) n^{2}} \cos ^{2} \omega
$$

$(\sin (\omega)$ and the relative refractive index, $n$, are introduced for experimental artefacts correction [50] - see Section 2.2.) The term $\sin (\omega) A_{\omega} / A_{\omega=\pi / 2}$ is the dichroic ratio $\left(\sin (\omega) A_{\omega}-A_{\omega=\pi / 2}\right.$ is sometimes named "linear dichroism" but the designation is more commonly used to mean the methodology itself instead of this experimental parameter). $\left\langle P_{2}\right\rangle$ obtained from Eq. (5) regards the absorption transition moment distribution. If the molecular axis and the transition moment are parallel, the molecular distribution function, has the same $\left\langle P_{2}\right\rangle$. If the angle between the transition moment and the molecular axis, $\xi$, is known, the experimentally calculated $\left\langle P_{2}\right\rangle$ can be converted into the molecular distribution (see Section 2.3).

The determination of $\left\langle P_{4}\right\rangle$ has been one of the targets of van der Meer and co-workers [8,21,47]. If the absorption and emission dipoles are parallel to the molecular symmetry axis, $\left\langle P_{4}\right\rangle$ can be calculated from (experimental set-up in Fig. 4):

$$
\frac{G I_{v h}}{f^{\prime}(\alpha) I_{v v}}=m \sin ^{2} \alpha+b
$$

( $\alpha$ is the angle depicted in Fig. 4).

$$
\begin{aligned}
m & =\frac{a}{n^{2}}, \\
a & =\frac{\left[\left(3 / 7\left\langle P_{4}\right\rangle+4 / 7\left\langle P_{2}\right\rangle-\left\langle P_{2}\right\rangle^{2}\right) w+\left\langle P_{2}\right\rangle^{2}-\left\langle P_{2}\right\rangle\right]}{c},
\end{aligned}
$$




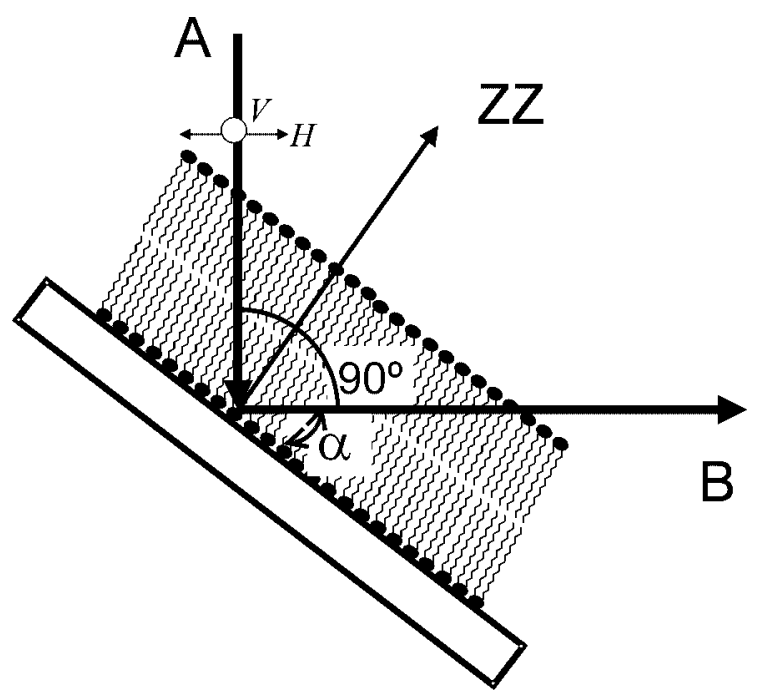

Fig. 4. Schematic representation of the experimental set-up for fluorescence LD studies in lipidic membranes (not drawn to scale). The excitation beam (A), with polarization "horizontal" $(H)$ or "vertical" ( $V$; perpendicular to the figure plane) in the lab frame, impinges on the quartz slide and supported bilayers (only one is represented for the sake of clarity). Fluorescence light collection (B) is carried out in a $90^{\circ}$ angle geometry The angle between them $(\omega)$ equals the one formed by the system director (ZZ) and the light polarization (B). Rotation of the quartz plate causes a variation in angle $\alpha$.

$$
\begin{aligned}
b & =\frac{1}{3} \frac{\left[1-\left(2 / 5+36 / 35\left\langle P_{4}\right\rangle+4 / 7\left\langle P_{2}\right\rangle-2\left\langle P_{2}\right\rangle^{2}\right) w-2\left\langle P_{2}\right\rangle^{2}+\left\langle P_{2}\right\rangle\right]}{c}, \\
c & =\frac{1}{3}\left[1+\left(\frac{4}{5}-\frac{4}{7}\left\langle P_{2}\right\rangle+\frac{27}{35}\left\langle P_{4}\right\rangle-\left\langle P_{2}\right\rangle^{2}\right) w+\left\langle P_{2}\right\rangle^{2}-2\left\langle P_{2}\right\rangle\right], \\
w & =\frac{\tau_{0}}{\tau_{0}+\tau} .
\end{aligned}
$$

The strong collision model was adopted while deriving these equations [47]. $\tau_{0}$ is a rotational relaxation time, which characterizes the anisotropic movement of the molecules. It can be interpreted as the time during which the transition moment has a particular, fixed orientation relative to the director. $G, f^{\prime}(\alpha)$ and $n$ are correction factors [47,50] (see Section 2.2). The subscripts in $I_{i j}$ refer to the position of the excitation $(i)$ or emission $(j)$ polarizers in the lab frame $(v$-vertical; $h$-horizontal).

As $\left\langle P_{2}\right\rangle$ is known from absorption experiments, $\left\langle P_{4}\right\rangle$ and $w$ can be obtained from the linear fit to the $G I_{v h} /\left(f^{\prime}(\alpha) \cdot I_{v v}\right) v s \cdot \sin ^{2} \alpha$ data. Alternatively, $w$ can be calculated from an independent experiment by measuring the steady-state fluorescence anisotropy, $r$, in unoriented lipidic vesicles and using:

$$
r=r_{0}\left[\left\langle P_{2}^{\prime}\right\rangle^{2}+w\left(1-\left\langle P_{2}^{\prime}\right\rangle^{2}\right)\right]
$$

( $r_{0}$ is the fluorescence anisotropy in the absence of external depolarising factors [27]). $\left\langle P_{2}^{\prime}\right\rangle$ refers to the second rank order parameter that would be obtained from fluorescence spectroscopy in unoriented vesicles, which is not necessarily equal to $\left\langle P_{2}\right\rangle$ obtained from Eq. (5); see Section 2.3. Assuming that $\left\langle P_{2}^{\prime}\right\rangle=\left\langle P_{2}\right\rangle, w$ can be calculated from Eq. (12). $\left\langle P_{4}\right\rangle$ can be calculated from both the slope and the intercept of $G I_{v h} /\left(f^{\prime}(\alpha) \cdot I_{v v}\right) v s \cdot \sin ^{2} \alpha$. Two estimates of $\left\langle P_{4}\right\rangle$ are attained for each $\left\langle P_{2}\right\rangle$. Concordance 
between both estimates can be used to assess data quality in a fast and simple, although crude, way. Furthermore, a specific value of $\left\langle P_{2}\right\rangle$ limits the range of possible $\left\langle P_{4}\right\rangle$ (Eq. (13) [21]).

$$
\left\langle P_{4}\right\rangle_{\min }=\left(\frac{35\left\langle P_{2}\right\rangle^{2}-10\left\langle P_{2}\right\rangle-7}{18}\right) \leqslant\left\langle P_{4}\right\rangle \leqslant\left(\frac{5\left\langle P_{2}\right\rangle+7}{12}\right)=\left\langle P_{4}\right\rangle_{\max } .
$$

\subsection{An approximate solution for $f(\psi) \sin (\psi)$ from $\left\langle P_{2}\right\rangle$ and $\left\langle P_{4}\right\rangle$}

Truncation of Eq. (4) to yield the first three terms (up to $P_{4}$ ) leads to a poor approximate description of $f(\psi)$. Frequently, the truncated function becomes negative, which is physically meaningless. The most common realistic approximation combines the application of the maximum entropy method with the Lagrange multipliers method [21]. In this approach, the approximated function is the one that maximizes the orientational entropy of the distribution, $S(f(\psi))$ :

$$
S(f(\psi))=\int_{0}^{\pi} \ln (\psi) \sin (\psi) f(\psi) \mathrm{d} \psi .
$$

In other words, the resulting distribution is the broadest possible from all the universe of distributions having that particular $\left(\left\langle P_{2}\right\rangle,\left\langle P_{4}\right\rangle\right)$ pair:

$$
f(\psi)=A \exp \left(\sum_{L} \lambda_{L} P_{L}(\cos \psi)\right)
$$

$A$ is a normalization constant and the $\lambda_{L}$ coefficients (Lagrange multipliers) are calculated from the accessible $\left\langle P_{L}\right\rangle$ values:

$$
\begin{aligned}
\left\langle P_{2}\right\rangle & =\int_{0}^{\pi} \sin \psi P_{2}(\cos \psi) f(\psi) \mathrm{d} \psi, \\
\left\langle P_{4}\right\rangle & =\int_{0}^{\pi} \sin \psi P_{4}(\cos \psi) f(\psi) \mathrm{d} \psi, \\
f(\psi) & =\frac{\exp \left(\lambda_{2} P_{2}(\cos \psi)+\lambda_{4} P_{4}(\cos \psi)\right)}{\int_{0}^{\pi} \sin \psi \exp \left(\lambda_{2} P_{2}(\cos \psi)+\lambda_{4} P_{4}(\cos \psi)\right) \mathrm{d} \psi} .
\end{aligned}
$$

It is interesting to have a global notion of the main features of $f(\psi)$ and their variation with $\left\langle P_{2}\right\rangle$ and $\left\langle P_{4}\right\rangle$. The readers are encouraged to see the elucidative Fig. 4 in [34]. Reference [46] has a more systematic description of peculiar cases:

(1) Total disorder. $\left\langle P_{2}\right\rangle=\left\langle P_{4}\right\rangle=0$.

(2) Total order. $\left\langle P_{4}\right\rangle=\left\langle P_{4}\right\rangle_{\min }$; the distribution is a delta function centered at $\psi^{\prime}$.

$$
\psi^{\prime}=\arccos \left(\frac{2}{3}\left\langle P_{2}\right\rangle+\frac{1}{3}\right)^{1 / 2}
$$




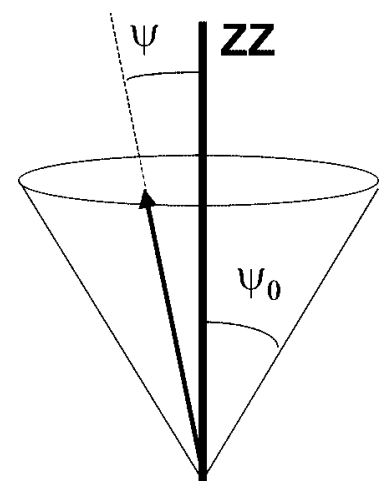

Fig. 5. If $\psi \leqslant \psi_{0}$, then the vector is restricted to wobbling in a cone.

(3) Free orientation in a cone of amplitude $\psi_{0}$. This case is analogous to a particle-in-a-box model. The molecules can adopt any orientation in the cone $\psi \leqslant \psi_{0}$ (Fig. 5) with equal probability:

$$
f(\psi)= \begin{cases}\left(2 \pi\left(1-\cos \psi_{0}\right)\right)^{-1}, & 0 \leqslant \psi \leqslant \psi_{0}, \\ 0, & \psi>\psi_{0} .\end{cases}
$$

and so:

$$
\begin{aligned}
& \left\langle P_{2}\right\rangle=\frac{1}{2} \cos \psi_{0}\left(1+\cos \psi_{0}\right) \\
& \left\langle P_{4}\right\rangle=\frac{1}{2} \cos \psi_{0}\left(1+\cos \psi_{0}\right)\left(7 \cos ^{2} \psi_{0}-3\right) .
\end{aligned}
$$

(4) Orientation determined by an angular potential. If $\lambda_{4}=0$ and $\lambda_{2} \neq 0, f(\psi)$ equals the one obtained when a Maier-Saupe potential (Eq. (23)) is used:

$$
U(\psi)=-k T \lambda_{2} P_{2}(\cos \psi) \text {. }
$$

(5) Bimodal orientation. If $\lambda_{2}=0$ and $\lambda_{4} \neq 0, f(\psi)$ has two maxima, at $\psi=0$ and $\psi=\pi / 2$.

(6) Double delta function. If $\left\langle P_{4}\right\rangle=\left\langle P_{4}\right\rangle_{\max }, f(\psi)$ is a double delta function at $\psi=0$ and $\psi=\pi / 2$.

\subsection{Experimental data correction for artefacts}

\subsubsection{Illuminated sample volume}

As the angle between the incident light beam and the sample surface ( $\omega$ in Fig. 3) decreases, the illuminated area of the sample surface increases. Therefore, absorbance increases. Reference [41] shows that this can be corrected with a sinus function; the term $\sin (\omega)$ in Eq. (5) has this purpose. Alternatively, $\sin (\omega) A_{\omega} / A_{\omega=\pi / 2}$ can be replaced by $A_{\omega, / /} / A_{\omega, \perp}$ where // and $\perp$ mean that the incident light polarization is parallel or perpendicular to the sample rotation axis, respectively. The illuminated area is equal in both cases and no correction is needed. However, two measurements are needed at each angle, which is time consuming and may turn data acquisition into a tedious task.

Fluorescence intensity measurements do not need to be corrected for this effect because relative quantities are considered; the effect is cancelled in the ratio $G I_{v h} /\left(f^{\prime}(\alpha) \cdot I_{v v}\right)$ (Eq. (6)). 


\subsubsection{Light scattering}

Langmuir Blodgett (LB) films usually are not turbid samples due to their thin thickness and macroscopic homogeneity. Semi-dehydrated lipid suspensions (lipidic multilayers; see Section 3.1) may, however result in turbid samples due to their thickness and/or inhomogeneous spreading of the lipid suspension over the solid substrate (e.g., quartz slide) surface during sample preparation. The effect of turbidity is to deviate the apparent absorption spectrum base line from zero, similarly to what is observed in vesicles suspensions [35]. Fluorescence intensity measurements are not so affected by light scattering.

Because turbidity can be minimized by "cautious" sample preparation, the main effort should be put on the experimental procedure rather than in corrective equations. Nevertheless, it is not always possible to eliminate scattering light effects in absorption measurements. Several papers have been published on light absorption on turbid media $[9,13,28,44,45]$. A correction method that simultaneously accounts for scattered light and for the flattening of the absorption spectra, resulting from inhomogeneous distribution of the chromophores can be deduced from the work of Bustamante and Maestre [9], as previously reported [49]. However, the simple empirical method of setting the base line as a segment connecting the lowest and highest energy extrema of the spectrum may lead to similar results (Fig. 6).

Finally, it should be stressed that lower wavelength radiation is more efficiently scattered, thus absorption spectra in the UV are more affected than spectra in the visible range.

\subsubsection{Emission monochromator efficiency dependence on polarization}

The monochromators transmission efficiency depends on the polarization of light relative to the diffraction grating. Fluorescence anisotropy measurements, for instance, need correction to account for this dependence [27]. Equation (6) considers the ratio of the two components of emitted polarized light when the sample is excited with "vertical" polarization (in the lab frame). This ratio must be corrected in a similar way, using $G=I_{h v} / I_{h h}$ (as before, the subscripts in $I_{i j}$ refer to the position of the excitation (i) or emission $(j)$ polarizers in the lab frame; $v$ - vertical; $h$ - horizontal). This is an intrinsic instrumental factor, which depends only on the emission monochromator transmittance for "vertical" and "horizontal" polarized light of a certain wavelength. Thus, $G$ can be determined with a homogenous isotropic sample, attaining more accurate results.

\subsubsection{Birefringence}

Kooyman et al. have shown that the birefringence correction is of the order of just a few percents due to the small optical anisotropy of macroscopically ordered lipid membranes [47]. This conclusion was later confirmed [8].

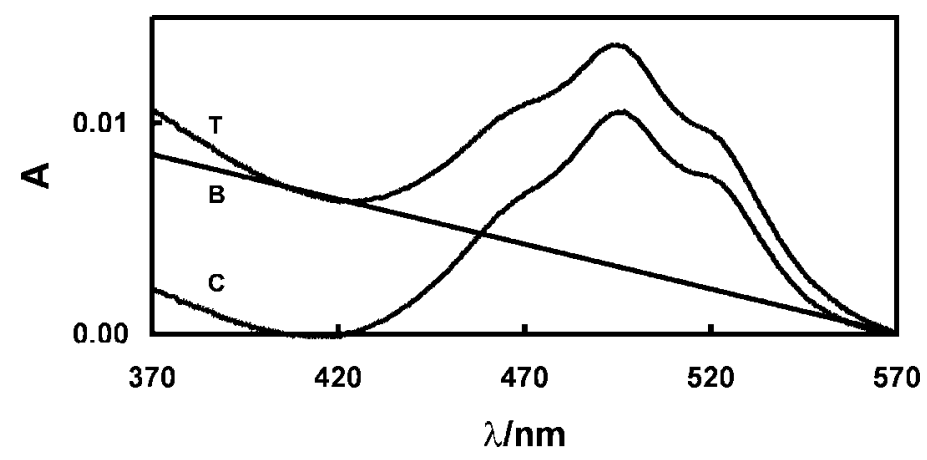

Fig. 6. Absorption spectrum of cyanine DIOC $_{6}$ in DPPC semi-dehydrated multilayers. The recorded spectrum $(\mathrm{T})$ at $\omega=18^{\circ}$ contains a contribution from light scattering. A base line was calculated (B) to account for turbidity effects (see text) and a corrected (C) spectrum was calculated by subtraction $(C=T-B)$. 


\subsubsection{Refraction}

Propagation of light passing through the interface of two isotropic media ( $i$ and $j$ ) with different refraction indexes $\left(n_{i}\right.$ and $\left.n_{j}\right)$ is described by Snell's law:

$$
\sin \theta_{i}=\frac{n_{j}}{n_{i}} \sin \theta_{j}
$$

$\left(\theta_{i}\right.$ and $\theta_{j}$ are the angles of the normal to the interface with propagation directions in media $i$ and $j$, respectively). Equation (5) includes a correction factor for this effect $\left(n=n_{j} / n_{i}\right.$, where $j=$ lipid and $i=$ air). In $\left\langle P_{4}\right\rangle$ determination from Eq. (6), no correction is needed for the polarization direction of the exciting light because excitation polarization is perpendicular to the director [47]. For fluorescence emission light with perpendicular polarization relative to excitation polarization, Snell's law relates propagation angles inside and outside the lipidic matrix. The slope in Eq. (6) has to be corrected for this effect (Eq. (7)).

\subsubsection{Reflection}

The transmission fraction upon incidence of light in an interface has to be accounted for. As the polarization relative to the plane of incidence affects the ratio $I_{v h} / I_{v v}$ (Eq. (6)), an angle dependent correction factor based on Fresnel's equations is needed [47]. The experimental ratio must be divided by the corrective fluorescence transmission ratio, $f^{\prime}(\alpha)$ (see Fig. 3 in [47]). Figure 7 shows a easy-to-use functional and empirical approximation to $f^{\prime}(\alpha)$ :

$$
f^{\prime}(\alpha)=1+\left(1.14 \times 10^{-2} \alpha\right)^{4.4144} \quad(\alpha \text { in degrees; } 0<\alpha<85) .
$$

Multiple reflections inside the sample is not a meaningful effect because it causes errors of less than $2 \%$ in the experimentally determined intensities [8].

\subsection{Main alternative methods to obtain $\left\langle P_{2}\right\rangle$ and $\left\langle P_{4}\right\rangle$ from optical spectroscopies}

\subsubsection{IR absorption spectroscopy}

Polarized ATR-FTIR (attenuated total reflection - Fourier transform infra-red) spectroscopy has been used to conclude on the orientation of biomolecules inserted in membranes, mainly proteins (for comprehensive reviews see [10] and [16]). The basic principles do not differ from the ones in UV-Vis. linear

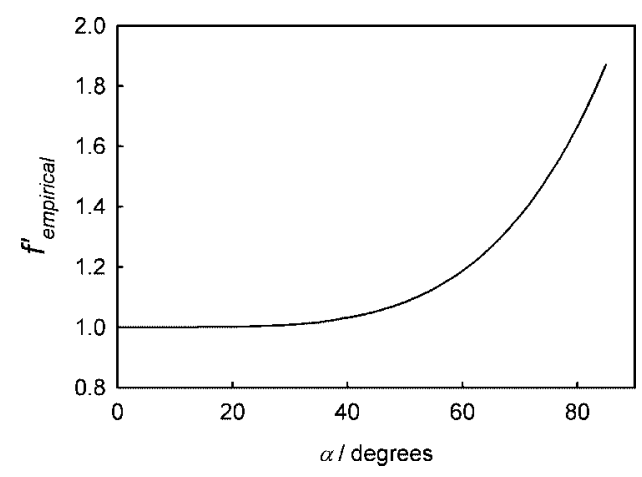

Fig. 7. Empirical approximation to the correction factor $f^{\prime}$, which accounts for the light reflection upon incidence in an interface [47]. The depicted function is a mere empirical description using Eq. (25), used for the sake of simplicity and functionality, and is valid in the range $0-85^{\circ}$. 
dichroism (maximal light absorption if the dipole transition moment is parallel to the electric field component of the incident light). Nevertheless, the use of ATR techniques has obvious advantages regarding signal/noise ratios. Although ATR techniques have been conjugated to UV-Vis. LD [42], this is not common. The main limitations of ATR-FTIR are: (1) only $\left\langle P_{2}\right\rangle$ can be calculated, (2) the dipole transition moment is not easily related to the molecular orientation in most cases (e.g., [24]), (3) previous knowledge on the sample thickness is needed, which can be quite complex to achieve, and (4) the superimposition of the vibrational bands of the lipidic matrix and foreign molecules may bias or even prevent $\left\langle P_{2}\right\rangle$ calculation. In spite of these limitations, ATR-FTIR is an alternative to UV-Vis. LD for low absorption samples. Limitation 1 may also be present in UV-Vis. LD because $\left\langle P_{4}\right\rangle$ determination is impossible with low fluorescence quantum yield molecules. The main strengths of ATR-FTIR are: (1) light scattering problems are insignificant and (2) sometimes, depending on the molecules to be studied, it is possible to simultaneously study these molecules and lipids.

\subsubsection{Fluorescence anisotropy decays}

Toptygin and Brand clearly reviewed some of the basic concepts underlying $\left\langle P_{2}\right\rangle$ and $\left\langle P_{4}\right\rangle$ determination from fluorescence anisotropy decays [14]. Fluorescence emission anisotropy, $r$, is defined as

$$
r=\frac{I_{/ /}-I_{\perp}}{I_{/ /}+2 I_{\perp}},
$$

where $I_{/ /}$and $I_{\perp}$ are the intensities of the polarization components parallel and perpendicular to the polarization of the excitation radiation, respectively. In its functional form, Eq. (26) reads:

$$
r=\frac{I_{v v}-G I_{v h}}{I_{v v}+2 G I_{v h}}
$$

( $G$ has the meaning mentioned before). The fluorescence anisotropy decay (ns time scale), $r(t)$, depends on the orientational order and dynamics during the fluorophore's excited state. At $t=0, r(t)$, is only dependent on intrinsic spectroscopic characteristics of the fluorophore. If the absorption and emission transition moments are parallel, $r(t=0)=r_{0}=0.4$ [27]. Fluorophores that follow orientational distributions that are not isotropic (as most molecules inserted in lipidic membranes) lead to limiting anisotropies, $r_{\infty}(r(t)$ in the limit $t \rightarrow \infty)$, different from zero (depolarisation is not complete). Equation (28) is commonly found in the literature.

$$
r_{\infty}=r_{0}\left\langle P_{2}^{\prime}\right\rangle^{2} .
$$

However, two very important assumptions are frequently overlooked:

(1) Equation (28) is only correct in the cases where the orientational distribution in the excited state equals the one in the ground state. A more general equation would be [32]:

$$
r_{\infty}=r_{0}\left\langle P_{2}^{\prime}\right\rangle\left\langle P_{2}^{\prime}\right\rangle^{*}
$$

(* denotes an excited state parameter).

(2) The local directors of the fluorophores are randomly oriented at the macroscopic level. Equations (28) and (29) hold true regardless of the orientation of the director axis relative to the membrane surface. No information can be obtained on the orientation of the director axis relative to the lipidic 
matrix [14]. Fluorescence anisotropy monitors distribution relative to the excitation photo-selection. The molecular orientation relative to the membrane cannot be known. This is the reason why $P_{2}^{\prime}$ is used in Eqs (28) and (29), as well as in Eq. (12); not to be confused with $P_{2}$, used in LD. Nevertheless, in practice, only three possible directors seem reasonable on empirical grounds: (i) the normal to the membrane plane (in fluid membranes this is the mean orientation of lipid acyl chains), (ii) an axis tilted relative to the membrane surface normal and parallel to the acyl chains in gel-phase membranes, or (iii) parallel to the membrane surface, when molecules adsorb on the surface or intercalate in between the two layers. It should be stressed that $\left\langle P_{L}\right\rangle(L=2,4,6, \ldots)$ relative to a certain director can be converted to $\left\langle P_{L}\right\rangle$ relative to other director [22]:

$$
\left\langle P_{L}\right\rangle_{\beta}=P_{L}(\cos \xi)\left\langle P_{L}\right\rangle_{\theta} .
$$

I.e. if the director axis is tilted by $\xi$ from the membrane normal, then the angular distribution relative to the director axis (angles $\beta$ ) is related to the angular distribution relative to the normal (angles $\theta$ ) by Eq. (30). In fact, Eq. (30) is only valid if $\xi$ and $\theta$ are independent [25].

An additional correction of $\left\langle P_{2}^{\prime}\right\rangle$, for the refraction of light at the lipid/water interface is, in practice, not meaningful [14].

The $r(t)$ decay from $r_{0}$ to $r_{\infty}$ is dependent on both $\left\langle P_{4}^{\prime}\right\rangle$ and the rotational diffusion rate of the fluorophore around an axis perpendicular to the transition dipole, $D_{\perp}$. Assuming that $D_{\perp}$ is the same in the whole bilayer, Zannoni et al. [11] attained a quantitative model for $r(t)$. The products of the Wigner rotation matrices and the Green functions obtained for the Smoluchovski rotational diffusion were considered. Van der Meer et al. [55] worked out simplified approximated equations, consisting of the sum of three exponentials plus a constant (Eq. (31)). Thus, $\left\langle P_{2}^{\prime}\right\rangle,\left\langle P_{4}^{\prime}\right\rangle$ and $D_{\perp}$ can be obtained as fitting parameters in unoriented vesicles (e.g. [12]).

$$
\begin{aligned}
r(t) & =r_{0}\left(\sum_{i=1}^{3} g_{i} \exp \left(-t / \phi_{i}\right)+g_{4}\right), \\
g_{1} & =\frac{1}{5}+\frac{2\left\langle P_{2}\right\rangle}{7}+\frac{18\left\langle P_{4}\right\rangle}{35}-\left\langle P_{2}\right\rangle^{2}, \\
g_{2} & =\frac{2}{5}+\frac{2\left\langle P_{2}\right\rangle}{7}-\frac{24\left\langle P_{4}\right\rangle}{35}, \\
g_{3} & =\frac{2}{5}-\frac{4\left\langle P_{2}\right\rangle}{7}+\frac{6\left\langle P_{4}\right\rangle}{35}, \\
g_{4} & =\left\langle P_{2}\right\rangle^{2}, \\
\phi_{1} & =g_{1} /\left[6 D_{\perp}\left(\frac{1}{5}+\frac{\left\langle P_{2}\right\rangle}{7}-\frac{12\left\langle P_{4}\right\rangle}{35}\right)\right], \\
\phi_{2} & =g_{2} /\left[12 D_{\perp}\left(\frac{1}{5}+\frac{\left\langle P_{2}\right\rangle}{14}+\frac{8\left\langle P_{4}\right\rangle}{35}\right)\right], \\
\phi_{3} & =g_{3} /\left[12 D_{\perp}\left(\frac{1}{5}-\frac{\left\langle P_{2}\right\rangle}{7}-\frac{2\left\langle P_{4}\right\rangle}{35}\right)\right] .
\end{aligned}
$$

Attempts were made to circumvent the limitation of not knowing the orientation of the director axis in lipidic matrix in vesicles suspensions. Toptygin and Brand [14] studied the influence of the refraction of 
light at the lipid-water interface in the radiative decay rate. The radiative rate depends on the refractive indexes inside and outside the membrane, as well as on the shape of the membrane, position of the fluorophore in the membrane and its orientation relative to the membrane. If the fluorophore can assume different orientations, the total fluorescence intensity decay will be non-exponential, although only small differences in the lifetime components are expected (their ratio is less than 1.5 [14]). The mean lifetime, $\langle\tau\rangle$, is related to $\left\langle P_{2}\right\rangle$ by:

$$
\langle\tau\rangle^{-1}=k_{\mathrm{nr}}+\gamma\left(1-\left\langle P_{2}\right\rangle\right) n_{0}+\gamma n_{l}^{-4} n_{0}^{5}\left(\left\langle P_{2}\right\rangle+\frac{1}{2}\right)
$$

( $k_{\mathrm{nr}}$ is the nonradiative decay rate, $\gamma$ is constant and $n_{0}$ and $n_{l}$ are the refractive indexes of bulk solvent and bilayer interior, respectively). $\left\langle P_{2}\right\rangle$ is usually calculated from experiments where $\langle\tau\rangle$ is measured as a function of $n_{0}$. The refractive index $n_{0}$ is usually increased by the addition of sucrose (e.g. [36]) and it is assumed that $k_{\mathrm{nr}}, \gamma, n_{l}$ and $\left\langle P_{2}\right\rangle$ do not vary.

$\left\langle P_{2}\right\rangle$ and $\left\langle P_{2}^{\prime}\right\rangle$ can be compared and their ratio be used to calculate $\xi$ (Eq. (30)) and then convert $\left\langle P_{4}^{\prime}\right\rangle$ in $\left\langle P_{4}\right\rangle$ (Eq. (30)).

\subsection{In search of whole-molecule information}

Spectroscopic techniques report information regarding only the molecular parts directly involved in state transition (e.g. chromophores in the UV-Vis. and active chemical bonds in IR spectroscopies). For most biomolecules and molecular probes, this is only a fraction of the whole molecular structure. Relating the chromophore orientation to the rest of the molecular conformation is very complex unless computational molecular simulation techniques are used. We have performed Brownian dynamics (BD) simulations with atomic level details to attain precise information on membrane probes structure and dynamics. BD allows spanning all the conformational space to produce long time trajectories, which give a more meaningful picture of processes occurring in membranes. A mean-field is used to simulate a bilayer environment, including contributions from (1) enclosing potential that emulates the finite thickness of the bilayer and also the hydrophilic nature of membrane interfaces, (2) the anchorage potential that places elements that interact favourably with membrane interfaces in their vicinity, and (3) the orientational potential, which emulates the anisotropic medium of a bilayer and the ordering effect induced by acyl chains in neighbouring molecules. The molecules understudy are regarded as an ensemble of friction elements moving in the environment of the phospholipidic bilayer. Further details on the model, energetics and simulation method can be found in [38] and [50]. Illustrative results will be presented in the next section.

\section{Experimental section}

\subsection{Sample preparations - macroscopically aligned lipidic bilayers}

Sample preparation is critical in LD techniques. Uncontrolled disorder in lipidic matrixes may lead to uncontrolled disorder in biomolecules orientational distribution. Bimodal distributions may reflect disorder induced by lipid packing, rather than being the "legitimate" distribution of the biomolecules themselves. Sample preparation can be carried out in two different ways: (1) the biomolecules 
or probes are previously mixed with the phospholipids in a macroscopically isotropic medium (solution or vesicle suspension) followed by membrane deposition, or (2) macroscopically aligned membrane systems are first formed and then incubated with the biomolecules or probes to be studied. Dufrêne and Lee [56] reviewed the anchoring of biomolecules to supported lipid bilayers. In 1999, Goorgmatigh et al. reviewed the preparation of supported lipidic multilayers for ATR-FTIR studies of proteins and lipids [16]. Here we will briefly focus on relevant recent contributions to sample preparation.

Langmuir-Blodgett (LB) films [48] are formed by sequential monolayer transfer of the lipid through an aqueous medium/air interface and are the most well studied supported membrane system. Very well organized lipidic mono and bilayers (rarely, trilayers) can be formed and the process controlled by a judicious choice of experimental conditions (pressure, transfer rate, etc.). The advent of atomic force microscopy (AFM) brought new insights into the structure of lipidic LB films at the molecular level, as well as their mechanical properties [57]. Holes are the most common defects [20,39,40] but IR transmission and reflection-absorption spectroscopies show that lipids are ordered like a crystalline phase [43]. The holes morphology and amount vary according to the kinds of lipids used [20]. A non-negligible infiltration of lipids into the aqueous sub-phase during lipid deposition has been reported [19] but it is not known if this is related to the holes occurrence.

Although LB films are well ordered and characterized, two severe limitations prevent their ubiquitous use in LD experiments: (1) three layers can be deposited on each side of the solid support (e.g., quartz slide), at the most, which implies a too short light-path in transmission studies involving molecules with weak oscillator strengths and/or low concentrations, and (2) only gel-phase membranes form LB bilayers, which is very restrictive. To circumvent the first limitation, hundreds of bilayers may be needed. Spontaneous deposition of suspended vesicles in a glass surface has been used for long (e.g. [1]) but it was only in the last three years that the process has been characterized at the molecular level. The sequence of events involved in the transition from attached liposomes to bilayer patches on hydrophilic and hydrophobic solid supports were visualized in situ by AFM [26]. The attached liposomes spread and flattened from the outer edges toward the center until the two membrane bilayers were stacked in the top of each other. The top bilayer then either rolls or slides over the bottom bilayer and the adjacent edges join to form a larger membrane patch. When bilayer patches resulting from newly adsorbed vesicles come in contact with previously formed supported bilayer patches, they fuse on the surface [5].

A modification to this procedure is commonly used in ATR-FTIR LD studies, several thousands of ordered bilayers being attained [16]. Multibilayer films are obtained by applying an aqueous lipid suspension onto one side of the ATR crystal and semi-drying it under a gentle stream of nitrogen. We, using quartz slides, followed the same procedure (Figs 1 and 8). Hydration water [23] remains in the sample; thickness homogeneity, however, may be critical and depends on the way semi-dryness is achieved: nitrogen stream should be gentle and spread evenly over the whole solid support surface. Kalb et al. [17] described an alternative technique to produce supported phospholipid bilayers on quartz, which uses a LB phospholipid monolayer over quartz as a substrate for vesicle fusion. Centrifugation deposition is other alternative (e.g. [52]).

\subsection{Instrumental adaptation}

As shown in Section 2, UV-Vis. LD resumes to measure absorption or emission intensities while varying the angle between the sample quartz slide and the incident light beam. Thus, instrumental adaptation consists in placing the sample slide on a goniometer (Fig. 9) and replacing the standard sample holder of the absorption spectrophotometer or spectrofluorimeter by the goniometer/slide set-up (Figs 10 and 11). 


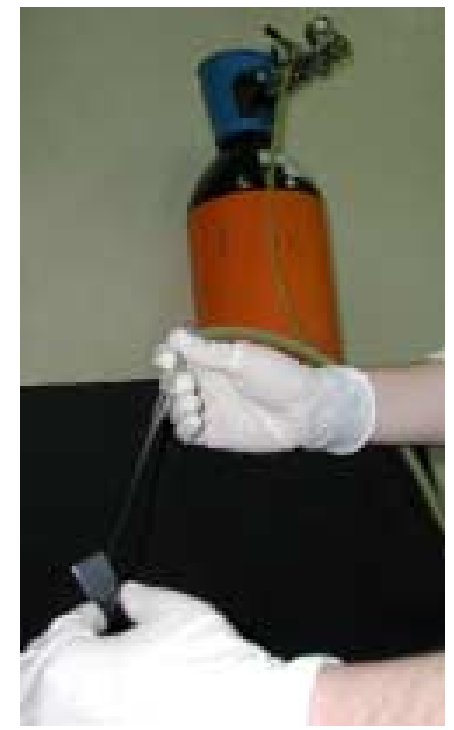

Fig. 8. Spontaneous multilayer deposition is a consequence of semi-dehydration under a gentle nitrogen flow. A Pasteur pipette was used to direct the nitrogen stream. It is very important that the lipid is evenly and homogenously spread over the quartz slide to minimize light scattering. (For the sake of picture contrast a very turbid sample was used.)

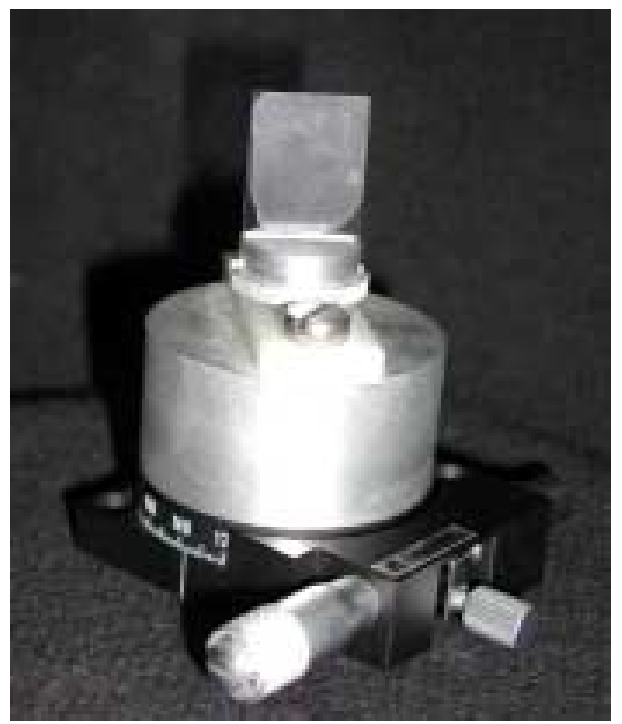

Fig. 9. The quartz slide was mounted on a goniometer using an aluminium homemade stand. Here the stand is shown in aluminium natural colour for better picture contrast. Likewise, a very turbid sample was used.

It should be stressed at this point that the absorption light path is very small (from six lipidic layers in LB films to hundreds of layers in semi-dehydrated lipidic suspensions). Moreover, polarizers may cause a substantial decrease in the intensity of incident light. Therefore, only very sensitive UV-Vis. absorption spectrophotometers can be used for LD experiments. This restriction is not so significant in spectrofluorimeters due to the intrinsic sensitivity of this technique. 


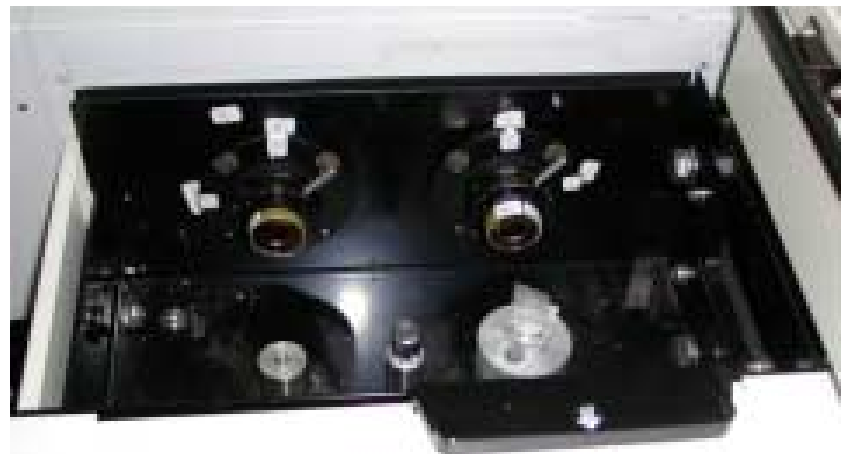

Fig. 10. The usual sample holder is replaced by the goniometer + stand system in the sample compartment of the UV-Vis. absorption spectrophotometer. The Glan-Thompson polarizers were adapted.

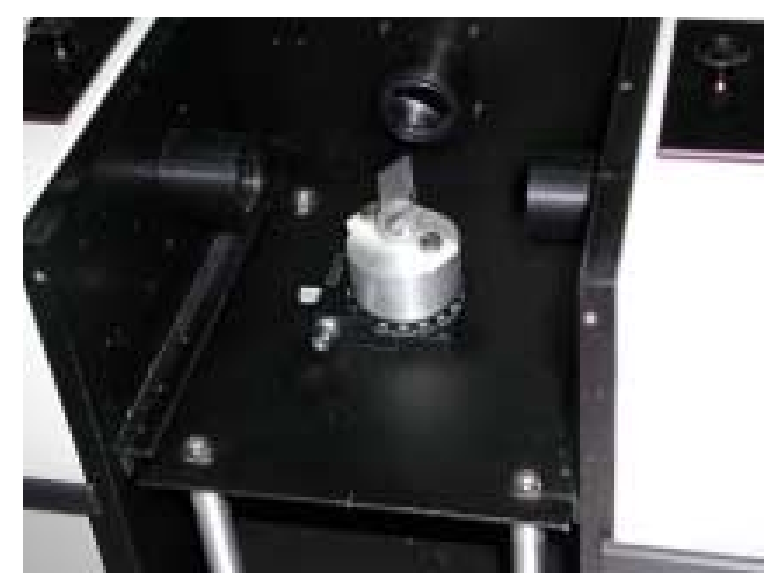

Fig. 11. The goniometer + stand system is placed in the center of the sample compartment of the spectrofluorimeter. The $90^{\circ}$ geometry for excitation/emission collection is clear.

\subsection{Apparatus and materials}

The detailed experimental procedures related to LB films and multibilayers formation were previously described in [49] and [50]. The following instruments were used: (1) NIMA (Coventry, UK) through, (2) Shimadzu UV-Vis. spectrophotometer (model UV-3101 PC) equipped with Glan-Thompson polarizers in home-made stands, and (3) SLM-Aminco 8100 spectrofluorimeter with double monochromators. The goniometer was purchased to Optosigma (Santa Ana, CA) and the quartz slides $(15 \times 25 \mathrm{~mm} ; 1 \mathrm{~mm}$ thick) were custom ordered from Precision Glass \& Optics (Santa Ana, CA). The slide stand (Fig. 9) was home-made.

Membrane probes were from Molecular Probes (Eugene, OR), except the cyanine (Acros Organics, Belgium); polyene antibiotics and sterols were from Sigma (St. Louis, MO); lipids were from Avanti Polar Lipids (Birgmingham, AL) and arachidic acid was from Analar (Germany). 
A

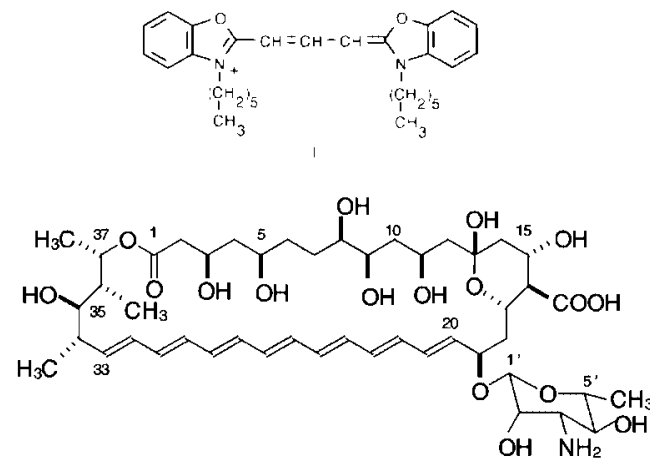

D

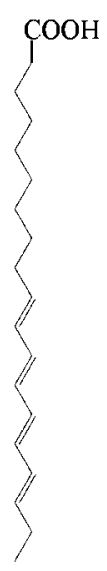

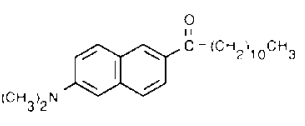

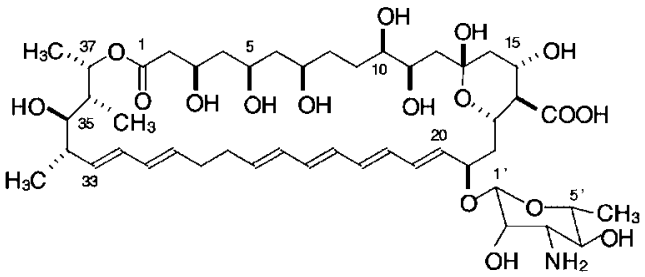

E

Fig. 12. UV-Vis. linear dichroism was applied to study the orientational distribution of several membrane probes (A-C) and polyene antibiotics $(\mathrm{D}, \mathrm{E})$. A - Cyanine dye $\mathrm{DIOC}_{6}, \mathrm{~B}$ - trans-parinaric acid, C - Laurdan, D - Amphotericin $\mathrm{B}$, and $\mathrm{E}-$ Nystatin $\mathrm{A}_{1}$. Transition moments are almost parallel to polyene chains in $\mathrm{B}, \mathrm{D}$ and $\mathrm{E}$, and inter-annular segment in $\mathrm{A}$; in $\mathrm{C}$, the transition moment is almost parallel to an imaginary segment connecting the derivatization groups in the ring system. Chemical intuition would favour orientations parallel to the lipid chains for B and C, and perpendicular for A.

\subsection{Results}

\subsubsection{Membrane probes}

UV-Vis. LD methodologies were applied to three membrane probes (Fig. 12): trans-parinaric acid $(t-\mathrm{PnA}$; a high quantum yield tetraene that reports phenomena both in gel-phase and liquid crystal membranes [7]), 3,3'-dihexyloxacarbocyanine $\mathrm{DIOC}_{6}$, a cyanine dye) and laurdan (a common polarity probe [54]). All of them have transition moments that can be considered parallel to the chromophore axis. Upon incorporation of $t$-PnA in LB films of either arachidic acid (multilayers) or dipalmitoylphosphatidylcholine (DPPC; three layers on each side of the quartz slide), spectral shifts occur, which are related to the different polarizabilities; vibrational progression alteration due to exciton interaction is possible in DPPC due to the high probe concentration (Fig. 13). Examples of excitation and emission spectra are shown in Fig. 14, regarding laurdan in DPPC multibilayers. The angular variation of the dichroic ratio is linear, as predicted by Eq. (5) (Fig. 15). $\left\langle P_{2}\right\rangle$ and $\left\langle P_{4}\right\rangle$ were determined as described previously (Eqs (5) and (6)), followed by calculation of $\lambda_{2}$ and $\lambda_{4}$ (Eqs (16) to (18)). The orientational density probability functions were depicted afterwards (Figs 16 and 17). The $t$-PnA distributions were compared to those obtained from BD simulation. The agreement was remarkable [38], validating the simulated distribution for a liquid-crystal phase $\left(L_{\alpha}\right)$ membrane (Fig. 16), which cannot be attained experimentally in LB films. Nevertheless, $t$-PnA has a mean orientation fairly parallel to the lipidic chains, as expected from chemical intuition. Moreover, the peak angle is similar to the tilt angle in DPPC gel [53]. Laurdan also follows the orientation expected from chemical intuition (Fig. 17). At variance, the cyanine does not follow the intuitive expectation (Fig. 17); it does not lye parallel to the membrane surface $\left(\psi=90^{\circ}\right)$. The influence of cyanines' aliphatic chain lengths on the orientational distribution is currently understudy. 


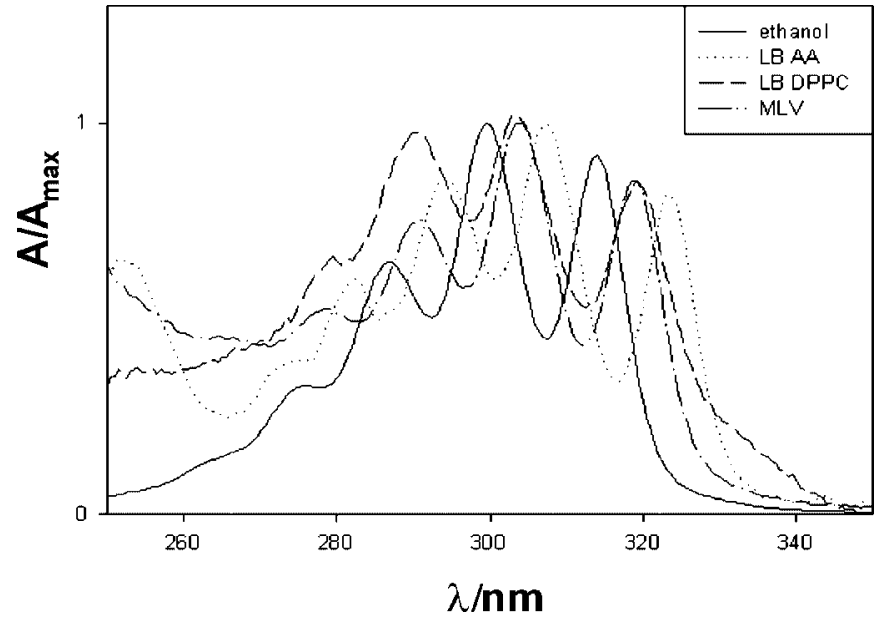

Fig. 13. Normalized electronic absorption spectra of $t$-PnA in LB multilayers of DPPC $\left(---, \omega=72^{\circ}\right)$, AA $\left(\cdots, \omega=72^{\circ}\right)$, aqueous suspension of multilamellar vesicles (MLV) of DMPC: DMPS $(3: 1)(-\cdot-\cdot-\cdot)$ and in homogeneous media (ethanol, -) [50].

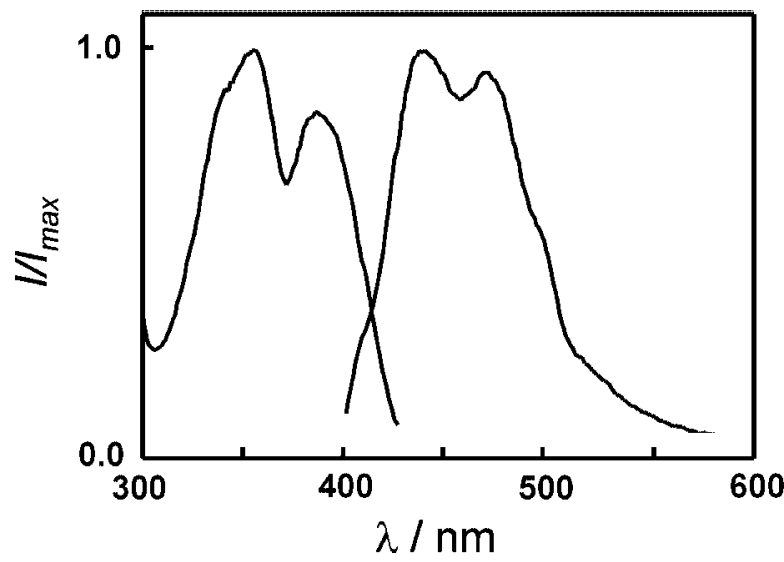

Fig. 14. Normalized excitation and emission fluorescence spectra of Laurdan in DPPC multibilayers at $\omega=45^{\circ}$ $\left(\lambda_{\mathrm{exc}}=350 \mathrm{~nm} ; \lambda_{\mathrm{em}}=440 \mathrm{~nm}\right.$; molar ratio DPPC $:$ Laurdan $=3: 1$; room temperature $)$.

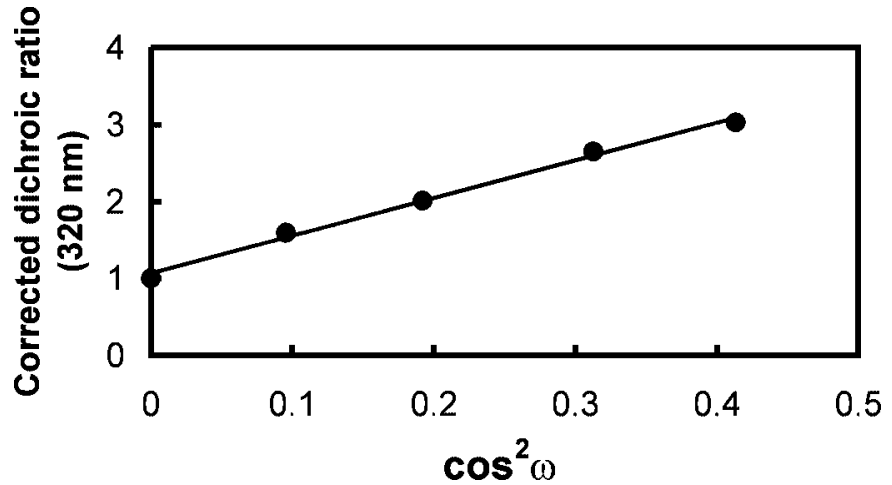

Fig. 15. Corrected dichroic ratio for $t$-PnA in LB DPPC films (three layers on each side of the quartz slide). 


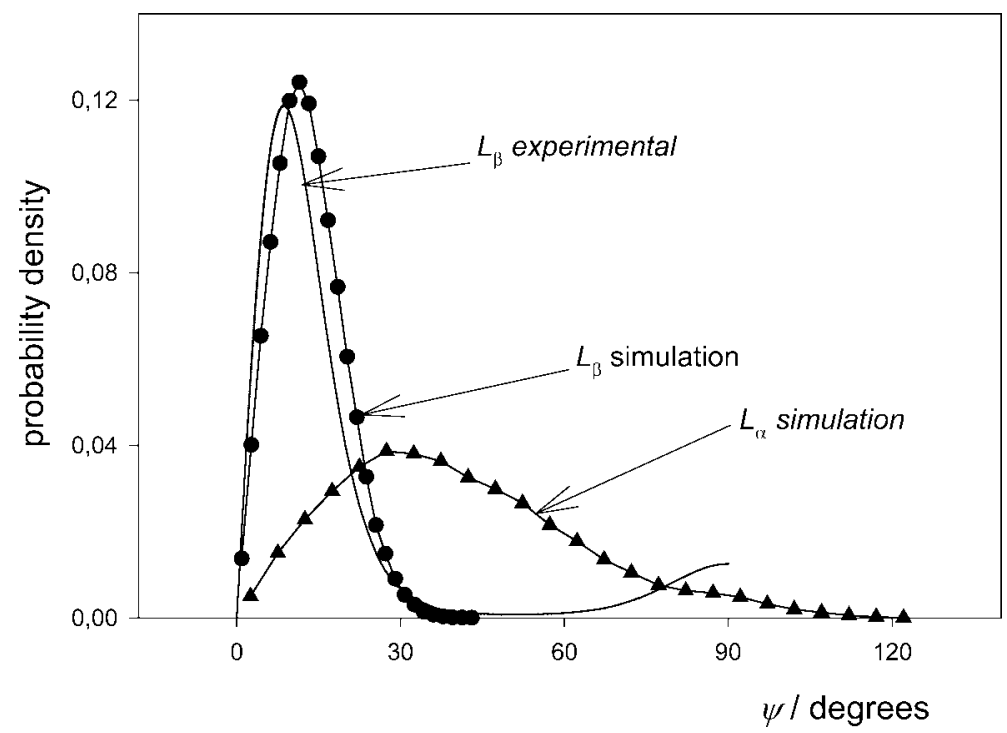

Fig. 16. Orientational density probability function of $t$-PnA in LB DPPC films (three layers on each side of the quartz slide) at room temperature ( $L_{\beta}$ phase). The distribution was compared to the one obtained by BD simulation [38]. The close agreement enabled deduction on other features of $t$-PnA conformation [38]. Moreover, it was possible to simulate the $L_{\alpha}$ lipidic phase, which cannot be attained by LB deposition techniques. The orientational distribution obtained with semi-dehydrated DPPC multilayers is similar to the one depicted for LB DPPC films at room temperature, which is evidence that semi-dehydrated multilayers resemble LB films in organization degree.

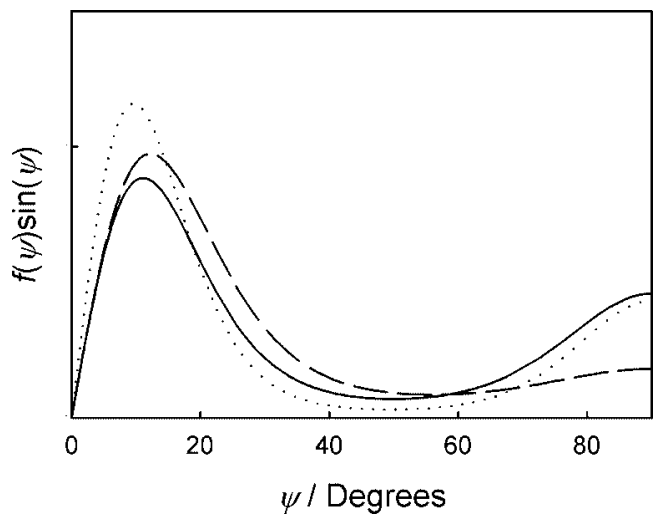

Fig. 17. Orientational distribution functions of laurdan $(-)$ in DPPC and cyanine DIOC 6 both in DPPC $(\cdots)$ and dilauroylphosphatidylcholine, DLPC (---) semi-dehydrated multibilayers at room temperature. Laurdan lies almost parallel to the bilayers' normal but cyanine does not distribute mainly parallel to the bilayers' surface as would be expected from chemical intuition. The phase of the lipid (DPPC is in the gel phase and DLPC in the fluid phase at room temperature) has little effect on the cyanine distribution. (Molar ratios lipid : cyanine $=20: 1$ and lipid : laurdan $=3: 1$.)

\subsubsection{Polyene antibiotics}

Polyene macrolide antibiotics, such as those depicted in Fig. 12, have long been used as therapeutic agents against systemic fungi infections. These molecules act at cellular membrane level, where they are thought to oligomerize and form pores [2]. Sterols are involved in pore formation. Although pores are usually depicted as parallel stacks of antibiotic molecules, perpendicular to the bilayer's surface, no direct evidence for this orientation was known until recently, when LD data was published [49]. At a first 


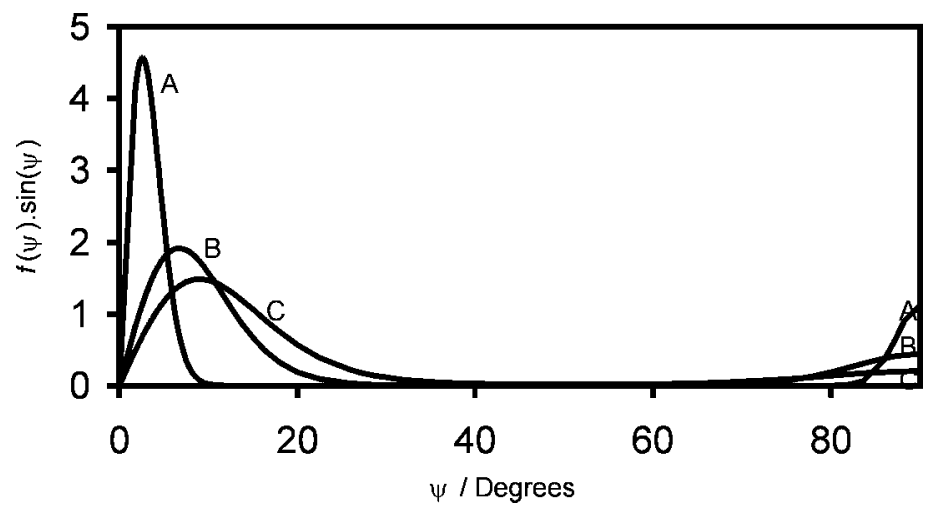

Fig. 18. Probability density functions for the polyene antibiotics in DPPC-cholesterol (A), AA-cholesterol (B) and DPPC (C) multilayers at room temperature [49]. Cholesterol interferes with the orientation of the antibiotics and plays a role in their biochemical mode of action.

glance, UV-Vis. LD could not be applied because polyene antibiotics having high absorptivity also have low fluorescence quantum yields (e.g., Amphotericin B - Fig. 12) and molecules having high fluorescent quantum yields, also have low absorptivities (e.g., Nystatin - Fig. 12). Nevertheless, Amphotericin B and Nystatin are remarkably similar in their chemical structure, the only difference being a double bond in the chromophore (Fig. 12). This detail leads to big photophysical differences among them while not affecting functional properties $[2,49]$. Thus, data obtained from both molecules was combined to calculate $\left\langle P_{2}\right\rangle$ and $\left\langle P_{4}\right\rangle$ [49]. $\left\langle P_{2}\right\rangle$ was obtained from the absorption data of Amphotericin B and $\left\langle P_{4}\right\rangle$ was obtained from the fluorescence emission of Nystatin. The resulting distributions are depicted in Fig. 18. The results show that the polyene antibiotics in the absence of cholesterol have an orientational distribution that is fairly broad and are almost collinear to the bilayer's normal. In the presence of cholesterol, the distribution becomes narrower. The sterol contributes to an increase in the order degree. This result is a clue that points to a role of the sterol in the antibiotics mode of action. The high order of the antibiotics orientation appearing upon cholesterol addition is compatible with the biochemical mode of action model that considers the formation of pore like structures composed of a packing of alternate sterols and antibiotics in a very organized array (see further discussion in [49]).

Although being a small contribution to the overall distribution, the small peaks at $90^{\circ}$ (present in both systems) deserve some attention. Several reasons can be pointed out:

(1) a fraction of the fluorophores lies parallel to the bilayers plane,

(2) a fraction of the bilayers has macroscopic defects and involves antibiotics molecules,

(3) the peaks are spurious and result from the data analysis method.

In practice, it is hard to distinguish between hypothesis (2) and (3) because $f(\psi) \sin (\psi)$ can only lead to bimodal distributions if one of the peaks is at $90^{\circ}$, i.e. a disordered subpopulation of antibiotics would lead to a biased distribution having a peak at $90^{\circ}$. To better ascertain the causes of such peak, measurements were also carried out in LB multilayer films composed of arachidic acid and cholesterol (Fig. 18). Arachidic acid multilayer deposited films are known to be well ordered and organized (see Section 3.1), therefore the contribution of disordered regions in the sample should be very small compared to films prepared by semi-dehydration. The presence of a bimodal distribution in this system points towards the exclusion of hypothesis (2). Although an option for hypothesis (1) or (3) cannot be firmly taken from the data, several researchers proposed that Amphotericin B could adsorb to the bilayer surface rendering the 
transition moment perpendicular to the system director axis (see references in [49]). If this is the case, then it can be concluded that the fraction of antibiotic molecules adsorbed to the bilayers is very small.

\section{Conclusions}

UV-Vis. LD techniques can be easily implemented, from both the instrumental and methodological points of view. Instrumental adaptation, sample preparation and data analysis are simple. $\left\langle P_{2}\right\rangle$ and $\left\langle P_{4}\right\rangle$ can be obtained for strongly absorbing and emitting molecules, enabling a quite straightforward estimate of the orientational density probability function. For low absorptivity molecules, $\left\langle P_{2}\right\rangle$ obtained from other LD techniques (e.g., ATR-FTIR LD) can be used, as long as the same system director is considered. UV-Vis. LD can complement and be complemented by other LD techniques. Combination of experimental and molecular simulation techniques help gain insight into the whole molecular structure in the membrane environment.

\section{Acknowledgements}

The authors thank Dr. Manuel Prieto (Technical University of Lisbon, Portugal) for valuable discussions, and Fundação para a Ciência e Tecnologia (Portugal) for funding.

\section{References}

[1] A.A. Brian and H.M. McConnell, Allogeneic stimulation of cytotoxic T cells by supported planar membranes, Proc. Natl. Acad. Sci. USA 81 (1984), 6159-6163.

[2] A. Coutinho and M. Prieto, Self-association of the polyene antibiotic Nystatin in dipalmitoylphosphstidylcholine vesicles: a time-resolved fluorescence study, Biophys. J. 69 (1995), 2541-2557.

[3] A. Holmgren, L.B-Å. Johansson and G. Lindblom, An FTIR linear dichroism study of lipid membranes, J. Phys. Chem. 91 (1987), 5298-5301.

[4] A. Rodger and B. Nordén, Circular Dichroism and Linear Dichroism, Oxford University Press, Oxford, 1997.

[5] A.S. Muresan and K.Y.C. Lee, Shape evolution of lipid bilayer patches adsorbed on mica: an atomic force microscopy study, J. Phys. Chem. B 105 (2001), 852-855.

[6] A. Saupe, The average orientation of solute molecules in nematic liquid crystals by proton magnetic resonance measurements and orientation dependent intermolecular forces, Mol. Cryst. 1 (1966), 527-540.

[7] B.S. Hudson and S.A. Cavalier, in: Studies of membrane dynamics and lipid-protein interactions with parinaric acid, L.M. Loew, ed., CRC Press, Boca Raton, FL, 1988, pp. 43-62.

[8] B.W. Van der Meer, R.P.H. Kooyman and Y.K. Levine, A theory of fluorescence depolarization in macroscopically ordered membrane systems, Chem. Phys. 66 (1982), 39-50.

[9] C. Bustamante and M.F. Maestre, Statistical effects in the absorption and optical activity of particulate suspensions, Proc. Natl. Acad. Sci. USA 85 (1988), 8482-8486.

[10] C. Vigano, L. Manciu, F. Buyre, E. Goormaghtigh and J.-M. Ruysschaert, Attenuated total reflection IR spectroscopy as a tool to investigate the stucture, otientation and tetiary structure changes in peptides and membrane proteins, Biopolymers 55 (2000), 373-380.

[11] C. Zannoni, A. Arcioni and P. Cavatorta, Fluorescence depolarization in liquid crystals and membrane bilayers, Chem. Phys. Lipids 32 (1983), 179-250.

[12] D.C. Mitchell and B.J. Litman, Molecular order and dynamics in bilayers consisting of highly polyunsaturated phospholipids, Biophys. J. 74 (1998), 879-891.

[13] D.S. Wiersma, P. Bartolini, A. Lagendijk and R. Righini, Localization of light in a disordered medium, Nature 390 (1997), 671-673.

[14] D. Toptygin and L. Brand, Determination of DPH order parameters in unoriented vesicles, J. Fluoresc. 5 (1995), 39-50. 
[15] E. Goormaghtigh and J.-M. Ruysschaert, Polarized attenuated total reflection infrared spectroscopy as a tool to investigate the conformation and orientation of membrane components, in: A Molecular Description of Biological Membranes by Computer Aided Conformational Analysis, Vol. I, R. Brasseur, ed., CRC Press, Boca Raton, 1990, pp. 285-329.

[16] E. Goormaghtigh, V. Rausseurs and J.-M. Ruysschaert, Attenuated total reflection spectroscopy of proteins and lipids in biological membranes, Biochim. Biophys. Acta 1422 (1999), 105-185.

[17] E. Kalb, S. Frey and L.K. Tamm, Formation of supported planar bilayers by fusion of vesicles to supported phospholipid monolayers, Biochim. Biophys. Acta 1103 (1992), 307-316.

[18] E.W. Thulstrup and J. Michl, Elementary Polarization Spectroscopy, John Wiley \& Sons, Inc., New York, 1989.

[19] G. Schwarz, G. Wackerbauer and S.E. Taylor, Partitioning of a nearly insoluble lipid monolayer into its aqueous subphase, Coll. Surf. A 111 (1996), 39-47.

[20] H.A. Rinia, R.A. Demel, J.P.J.M. van der Eerden and B. de Kruijff, Blistering of Langmuir-Blodgett bilayers containing anionic phospholipids as observed by atomic force microscopy, Biophys. J. 77 (1999), 1683-1693.

[21] H. Pottel, W. Herreman, B.W. van der Meer and M. Ameloot, On the signification of the fourth-rank orientational order parameter of fluorophores in membranes, Chem. Phys. 102 (1986), 37-44.

[22] I.M. Ward, Determination of molecular orientation by spectroscopic techniques, Adv. Polymer Sci. 66 (1985), 81-115.

[23] I.R. Miller and D. Bach, Organization of water molecules by adhering to oriented layers of dipalmitoylphosphatidyl serine in the presence of varying concentrations of cholesterol, Biochim. Biophys. Acta 1468 (2000), 199-202.

[24] I.T. Arkin, K.R. MacKenzie and A.T. Brünger, Site-directed dichroism as a method for obtaining rotational and orientational constraints for oriented polymers, J. Am. Chem. Soc. 119 (1997), 8973-8980.

[25] J.I. Cail, D.J.R. Taylor, R.F.T. Stepto, M.G. Brereton, R.A. Jones, M.E. Ries and I.M. Ward, Computer simulation studies of molecular orientation in polyethylene networks: orientation functions and the Legendre addition theorem, Macromolecules 33 (2000), 4966-4971.

[26] J. Jass, T. Tjärnhage and G. Puu, From liposomes to supported, planar bilayer structures on hydrophilic and hydrophobic surfaces. An atomic force microscopy study, Biophys. J. 79 (2000), 3153-3163.

[27] J.R. Lakowicz, Principles of Fluorescence Spectroscopy, 2nd edn, Kluwer Academic/Plenum Press, New York, 1999, Ch. 10.

[28] J. Wu, M.S. Feld and R.P. Rava, Analytical model for extracting intrinsic fluorescence in turbid media, Applied Optics 32 (1993), 3585-3595.

[29] L.B.-A. Johansson, A. Davidsson, G. Lindblom and B. Norden, Linear dichroism as a tool for studying molecular orientation in membrane systems. Order parameters of guest molecules from linear dicroism and nuclear magnetic resonance, J. Phys. Chem. 82 (1978), 2604-2609.

[30] L.B.-Å. Johansson, Analysis and application of linear dichroism on membranes, J. Chem. Soc. Faraday Trans. I 81 (1985), $1375-1388$

[31] L.B.- $\AA$. Johansson, G. Lindblom, A. Wieslander and G. Arvidson, Orientation of $\beta$ carotene and retinal in lipid bilayers, FEBS Lett. 128 (1981), 97-99.

[32] L.B.-Å. Johansson, Order parameters of fluorophores in ground and excited states. Probe molecules in a lyotropic liquid crystal, Chem. Phys. Lett. 118 (1985), 516-521.

[33] L.B.-A. Johansson, T. Vallmark and G. Lindblom, Polarized-light spectroscopic study of indocarbocyanine dyes solubilized in amphiphile aggregates, J. Chem. Soc. Faraday Trans. I 81 (1985), 1389-1400.

[34] M.A. Bos and J.M. Kleijn, Determination of the orientation distribution of adorbed fluorophores using TIRF. I. Theory, Biophys. J. 68 (1995), 2566-2572.

[35] M.A.R.B. Castanho, N.C. Santos and L.M. Loura, Separarting the turbidity spectra of vesicles from the absorption spectra of membrane probes and other chromophores, Eur. Biophys. J. 26 (1997), 253-259.

[36] M.M.G. Krishna and N. Periasamy, Fluorescence of organic dyes in lipid membranes: Site of solubilization and effects of viscosity and refractive index on lifetimes, J. Fluoresc. 8 (1998), 81-91.

[37] M.P. Heyn, Determination of lipid order parameters and rotational correlation times from fluorescence depolarization experiments, FEBS Lett. 108 (1979), 359-364.

[38] M.X. Fernandes, J. García de la Torre and M.A.R.B. Castanho, A Brownian dynamics simulation of na acyl chain and a trans-parinaric acid molecule confined in a phospholipid bilayer in the gel and liquid-crystal phases, J. Phys. Chem. B 104 (2000), $11579-11584$.

[39] N.C. Santos, E. Ter-Ovanesyan, J.A. Zasadzinski and M.A.R.B. Castanho, Reconstitution of phospholipid bilayer by an atomic force microscope tip, Biophys. J. 75 (1998), 2119-2120.

[40] N.C. Santos, E. Ter-Ovanesyan, J.A. Zasadzinski, M. Prieto and M.A.R.B. Castanho, Filipin-induced lesions in planar phospholipid bilayers imaged by atomic force microscopy, Biophys. J. 75 (1998), 1869-1873.

[41] N.C. Santos and M.A.R.B. Castanho, Teaching light scattering spectroscopy: The dimension and shape of Tobacco Mosaic Virus, Biophys. J. 71 (1996), 1641-1646.

[42] P.L. Edmiston, J.E. Lee, L.L. Wood and S.S. Saavedra, Dipole orientation distributions in Langmuir-Blodgett films by planar waveguide linear dichroism and fluorescence anisotropy, J. Phys. Chem. 100 (1996), 775-784. 
[43] P. Lozano, A.J. Fernández, J.J. Ruiz, L. Camacho, M.T. Martín and E. Muñoz, Molecular organization of LB multilayers of phospholipid and mixed phospholipid/viologen by FTIR spectroscopy, J. Phys. Chem. B (2002), in press (available on-line).

[44] P. Wittung, J. Kajanus, M. Kubista and B.G. Malmström, Absorption flettening in the optical spectra of liposomeentrapped substances, FEBS Lett. 352 (1994), 37-40.

[45] R.F. Bonner, R. Nossal, S. Havlin and G.H. Weiss, Model for photon migration in turbid biological media, J. Opt. Soc. Am. A 4 (1987), 423-432.

[46] R.M. Martinez, Estructura y dinamica de las biomembranes observada por espectroscopia de fluorescência com resolucion temporal: la membrana plasmatica de plaqueta humana, Ph.D. Dissertation, Universidad Autonoma de Madrid, 1989.

[47] R.P.H. Kooyman, Y.K. Levine and B.W. Van der Meer, Measurement of second and fourth rank order parameters by fluorescence polarization experiments in a lipid membrane system, Chem. Phys. 60 (1981), 317-326.

[48] G.G. Roberts (ed.), Langmuir Blodgett Films, Plenum Press, NY, 1990.

[49] S. Lopes and M. Castanho, Revealing the orientation of Nystatin and Amphotericin B in lipidic multilayers by UV-Vis. linear dichroism, J. Phys. Chem. B (2002), 7278-7282.

[50] S. Lopes, M.X. Fernandes, M. Prieto and M. Castanho, Orientational order of the membrane probe polyene fatty acid trans-parinaric acid in Langmuir-Blodgett multilayerfilms, J. Phys. Chem. B 105 (2001), 562-568.

[51] S. Tristram-Nagle, R. Zhang, R.M. Suter, C.R. Worthington, W.-J. Sun and J.F. Nagle, Measurement of chain tilt angle in fully hydrated bilayers of gel phase lecithins, Biophys. J. 64 (1993), 1097-1109.

[52] S.W. Lin and R.A. Mathies, Orientation of the protonated retinal Schiff base group in bacteriorhodopsin from absorption linear dichroism, Biophys. J. 56 (1989), 653-660.

[53] T. Hasegawa, Y. Ushiroda, M. Kawaguchi, Y. Kitazawa, M. Nishiyama, A. Hiraoka and J. Nishijo, UV absorption spectroscopic analysis of the molecular orientation of a drug penetrated into a DPPC membrane, Langmuir 12 (1996), 1566-1571.

[54] T. Parasassi, E.K. Krasnowska, L. Bagatolli and E. Gratton, Laurdan and Prodan as polarity-sensitive fluorescent membrane probes, J. Fluoresc. 8 (1998), 365-373.

[55] W. van der Meer, H. Pottel, W. Herrennan, M. Ameloot, M. Hendrickx and H. Schröder, Effect of orientational order on the decay of the fluorescence anisotropy in membrane suspensions, Biophys. J. 46 (1984), 515-523.

[56] Y.F. Dufrêne and G.U. Lee, Advances in the characterization of supported lipid films with the atomic force microscope, Biochim. Biophys. Acta 1509 (2000), 14-41.

[57] Y. Oishi, T. Umeda, M. Kuramori and K. Suehiro, Mechanical properties of a Langmuir-Blodgett film measured by atomic force microscopy, Langmuir 18 (2002), 945-947.

[58] Y. Shimoyama, L.E.G. Eriksson and A. Ehrenherg, Molecular motion and order in oriented lipid multibilayer membranes evaluated by simulations of spin label ESR spectra, Biochim. Biophys. Acta 508 (1978), 213-235. 


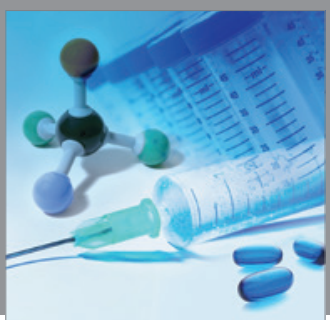

International Journal of

Medicinal Chemistry

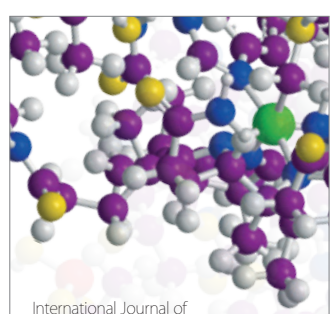

Carbohydrate Chemistry

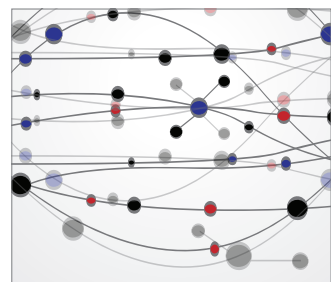

The Scientific World Journal
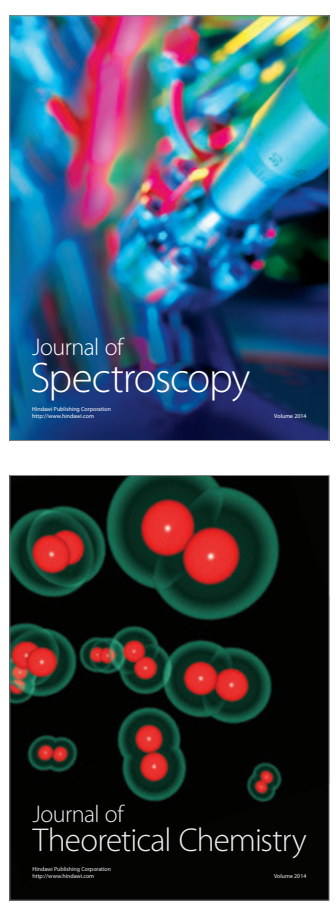
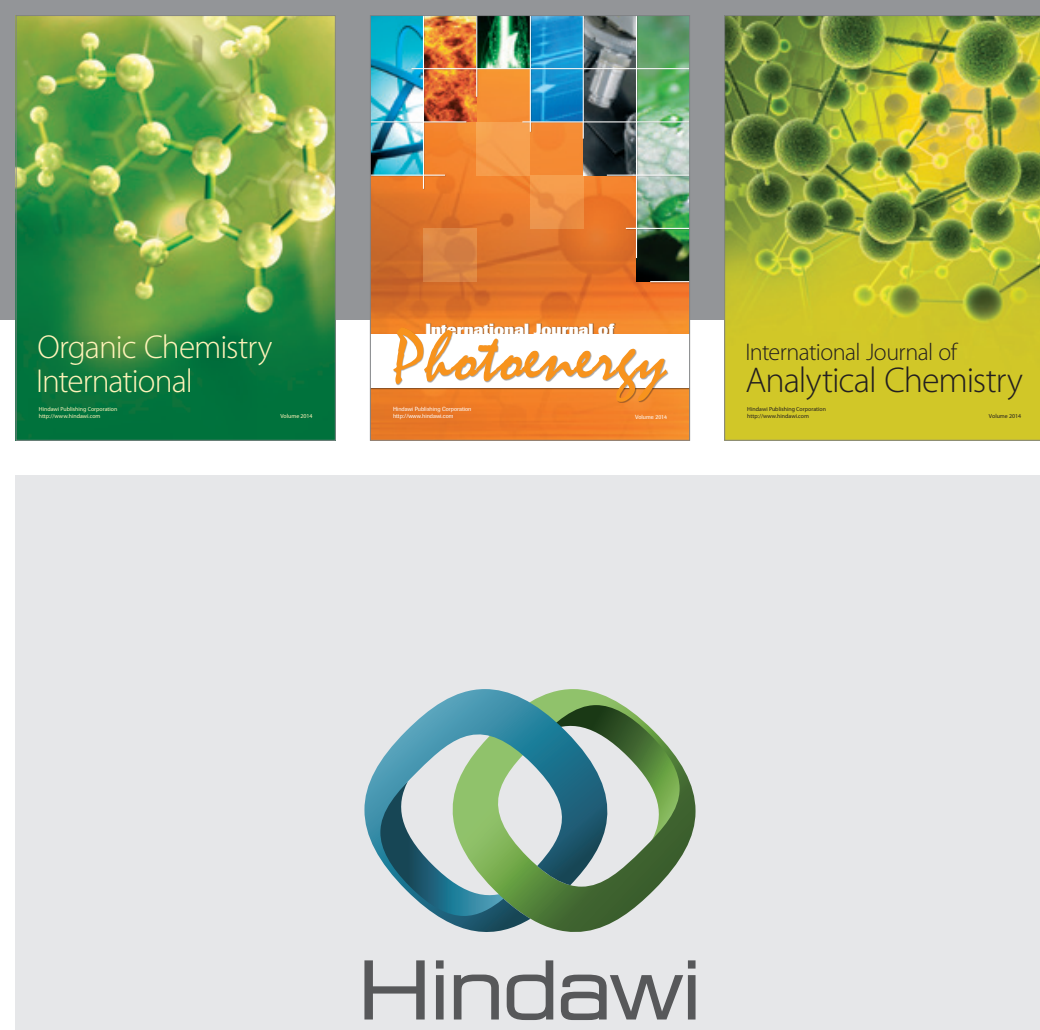

Submit your manuscripts at

http://www.hindawi.com
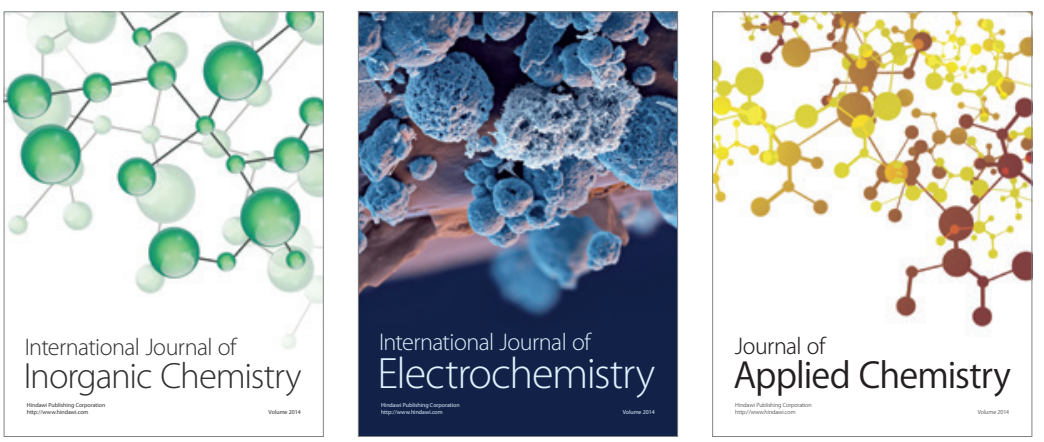

Journal of

Applied Chemistry
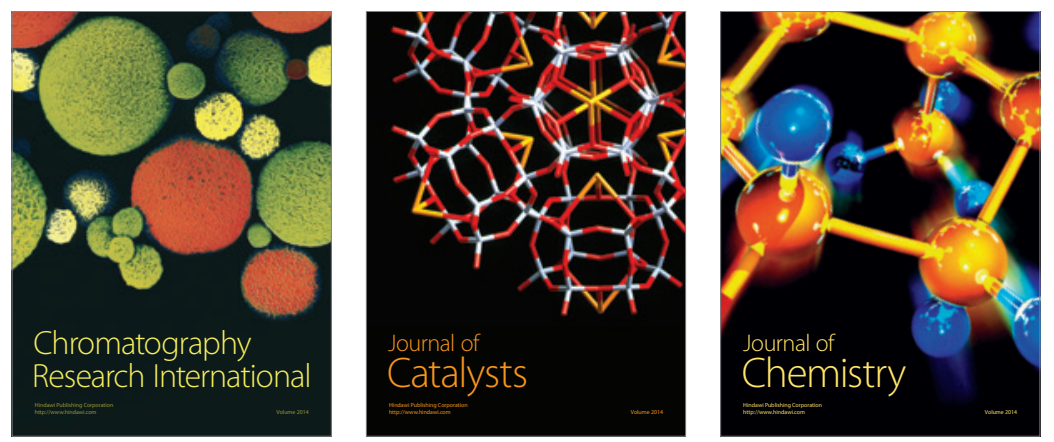
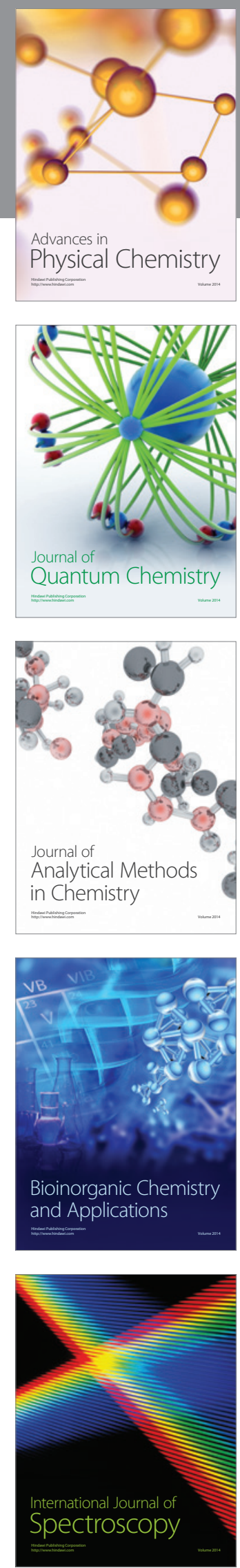